\title{
Some General Results On Quantile Functions For The Generalized Beta Family
}

\author{
Dreamlee Sharma *and Tapan Kumar Chakrabarty \\ Department of Statistics, North Eastern Hill University, Meghalaya, India.
}

(Received: 9 May 2017; Accepted: 21 November 2017)

\begin{abstract}
In this article we study and obtain some general results on the quantile functions for the generalized beta family and the family of beta generated distributions. Having described the standardization rule, we have derived the quantile function of the 5 parameter generalized beta family of distributions [21, 22]. Further, quantile rules for distributional model building have been applied to generate quantile functions of several known and unknown distributions. Attempts have been made to obtain and study the quantile functions of size biased generalized beta distributions and generalized beta generated distributions. Finally, we have applied the proposed results to simulated as well as real datasets.
\end{abstract}

Keywords Quantile function, Inverse regularized beta function, Q-transformation, p-transformation, beta generated quantile function.

\section{AMS 2010 subject classifications $60 \mathrm{E} 05,62 \mathrm{~F} 10$}

DOI: $10.19139 /$ soic.v5i4.312

\section{Introduction}

A quantile is simply the value that corresponds to a specified proportion of a sample or population. The quantile function $(\mathrm{QF})$ is thus a function that gives the quantile values for all probabilities $p, 0 \leq p \leq 1$ of a distribution. Mathematically, the general form of the quantile function of a distribution is defined as

$$
\inf \left\{X \mid F_{X}(X) \geq p\right\}=F_{X}^{-1}(p)
$$

Thus, if $F(Y)$ is the distribution function of $\mathrm{Y}$ such that $F(Y)=p$ then $Y=F^{-1}(p)=Q(p)$ is the corresponding quantile function.

The function $q(p)=\frac{d}{d p} Q(p)$ is called the quantile density function and it provides an alternative way to plot the probability density function ( $p . d . f$.) corresponding to quantile functions [30]. If we let $p$ take the values, say $p_{i}=0.01,0.02, \ldots, 0.99$ and plot the points $(Q(p), 1 / q(p))$, then we get the plot of points $(x, f(x))$, i.e., the plot of the $p . d . f$. of $x$. The quantity $1 / q(p)=f_{p}(p)$ is called the $p-P D F$ or density quantile function of a distribution. Thus we can obtain plots of the p.d.f. of a distribution entirely from the

\footnotetext{
${ }^{*}$ Correspondence to: Dreamlee Sharma (Email: dreamleesharma@yahoo.in). Department of Statistics, North-Eastern Hill
} University, Shillong - 793022, Meghalaya, India.

ISSN 2310-5070 (online) ISSN 2311-004X (print)

Copyright (c) 2017 International Academic Press 
quantile function and its derivative, without having to invert $Q(p)$ to get $F(x)$. For a detailed information about quantile modelling one can refer to [12].

Lemma 1

(The standardization rule) The quantile function of any distribution can be written in the general form

$$
Q(p)=\lambda+\eta S(p ; \boldsymbol{\alpha})
$$

where, $\lambda$ is the location parameter, $\eta$ is the scale parameter and $\boldsymbol{\alpha}$ is a vector of shape parameters and $S(p, \boldsymbol{\alpha})$ is the quantile function of the standard distribution.

Proof

Let $Y$ be a continuous random variable, then the location-scale model for $Y$ is given by $Y=\lambda+\eta Y_{0}$, where $Y_{0}$ has the standard distribution $F_{0}(Y)$. Then $F(Y)=F_{0}\left(\frac{Y-\lambda}{\eta}\right)$.

Let, $F(Y)=p$, then

$$
\begin{aligned}
\frac{Y-\lambda}{\eta} & =F_{0}^{-1}(p) \\
\text { or } \quad Y & =\lambda+\eta F_{0}^{-1}(p)
\end{aligned}
$$

By definition, $Y=F^{-1}(p)=Q(p)$, so that equation (1) becomes,

$$
Q(p)=\lambda+\eta Q_{0}(p)
$$

where $Q_{0}(p)$ is the standard quantile function.

Now, the standard distribution $F_{0}(Y)$ is free from the location and scale parameter, but it may contain parameter(s) that control the shape of the distribution, so that the standard quantile function, $Q_{0}(p)$ may contain shape parameter(s) as well. Thus, including the shape parameters, $\boldsymbol{\alpha}$ in the model (3) we have,

$$
Q(p)=\lambda+\eta S(p, \boldsymbol{\alpha})
$$

which is the general form of any quantile function.

\subsection{Some Definitions and Rules}

There are some quantile operations, rules and transformations which when applied to QF give valid QF and so can be used for distributional model building. For details one can refer to [12]. Table 1 summarizes these transformations.

Using the Q-transformation rule we can define the uniform transformation rule. If $U$ has a uniform distribution then the variable $X$, where $x=Q(u)$, has a distribution with quantile function $Q(p)$. Thus data and distributions can be visualized as being generated from the uniform distribution by the transformation $Q($.$) , where Q(p)$ is the quantile function.

\section{An introduction to beta quantile function}

Beta distribution, a family of continuous probability distributions defined on the interval $[0,1]$ is denoted by the probability density function (p.d.f) and quantile function given respectively in (4) and (5).

$$
g(y)=\frac{1}{B(\alpha, \beta)} y^{\alpha-1}(1-y)^{\beta-1}, \quad 0 \leq y \leq 1
$$


Table 1. Construction rules for distributional model building

\begin{tabular}{|c|c|c|c|}
\hline $\mathrm{QF}$ of variable $X$ & Operation & Resulting QF $Q(p)$ & $\begin{array}{l}\text { Transformation } \\
\text { and Resulting variable }\end{array}$ \\
\hline$Q(p)$ & Reflection rule & $-Q(1-p)$ & QF of $-X$ \\
\hline$Q(p)$ & Reciprocal rule & $\frac{1}{Q(1-p)}$ & QF of $\frac{1}{X}$ \\
\hline$Q_{1}(p), Q_{2}(p)$ & Addition rule & $Q(p)=Q_{1}(p)+Q_{2}(p)$ & $Q(p)$ is a valid $\mathrm{QF}$ \\
\hline$Q_{1}(p), Q_{2}(p)$ & Weighted sum rule & $Q(p)=w Q_{1}(p)+(1-w) Q_{2}(p)$ & $Q(p)$ is a valid $\mathrm{QF}$ \\
\hline$Q_{1}(p), Q_{2}(p)>0$ & Multiplication rule & $Q(p)=Q_{1}(p) * Q_{2}(p)$ & $Q(p)$ is a valid $\mathrm{QF}$ \\
\hline$Q(p)$ & $\begin{array}{l}\text { Q-transformation } \\
\text { rule for non } \\
\text { - decreasing } \\
\text { function } T_{1}(X) \text { of } X\end{array}$ & $T_{1}(Q(p))$ & QF of $T_{1}(X)$ \\
\hline$Q(p)$ & $\begin{array}{l}\text { Q-transformation } \\
\text { rule for non } \\
\text { - increasing } \\
\text { function } T_{2}(X) \text { of } X\end{array}$ & $T_{2}(Q(1-p))$ & $\mathrm{QF}$ of $T_{2}(X)$ \\
\hline$Q(p)$ & $\begin{array}{l}\text { p-transformation } \\
\text { rule for non } \\
\text { - decreasing } \\
\text { function } H_{1}(p) \text { of } p\end{array}$ & $Q\left(H_{1}(p)\right)$ & p-transformation of $Q(p)$ \\
\hline$Q(p)$ & $\begin{array}{l}\text { p-transformation } \\
\text { rule for non } \\
\text { - increasing } \\
\text { function } H_{2}(p) \text { of } p\end{array}$ & $Q\left(1-H_{2}(p)\right)$ & reversed p-transformation of $Q(p)$ \\
\hline
\end{tabular}

$$
Q_{B}(p)=I_{p}^{-1}(\alpha, \beta)
$$

where $\alpha>0$ and $\beta>0$ are the two shape parameters of the distribution and $I_{p}^{-1}(\alpha, \beta)$ is the inverse regularized beta function. Closed form expression for the beta QF exists only for the following three cases

1. For $\alpha=1, Q(p)=1-(1-p)^{\frac{1}{\beta}}$

2. For $\beta=1, Q(p)=p^{\frac{1}{\alpha}}$

3. For $\alpha=\beta=1, Q(p)=p$

Lemma 2

Let $Y$ be a beta random variable (r.v.) with p.d.f as given in (4). If we replace the random variable $Y$ by an arbitrary cumulative distribution function (c.d.f.) $F($.), then the quantile function (QF) for the transformed variable is given by

$$
Q_{F}(p)=Q_{1}\left(I_{p}^{-1}(\alpha, \beta)\right)
$$

where $Q_{1}($.$) is the quantile function corresponding to F($.$) .$ 
Proof

If $Y$ is a beta $r . v$. then its $c . d . f$. is given by

$$
\begin{aligned}
F_{b}(y) & =\int_{0}^{y} \frac{1}{B(\alpha, \beta)} x^{\alpha-1}(1-x)^{\beta-1} d x \\
& =I_{y}(\alpha, \beta)
\end{aligned}
$$

where $I_{y}(\alpha, \beta)$ is the regularized beta function.

Now, since $Y$ is replaced by an arbitrary distribution function $F(x)$, say, then the density function corresponding to $F(x)$ is

$$
g_{F}(x)=\frac{1}{B(\alpha, \beta)} f(x)[F(x)]^{\alpha-1}(1-F(x))^{\beta-1}, \quad 0 \leq F(X) \leq 1
$$

where $f(x)$ is the derivative of $F(x)$ and therefore is the corresponding p.d.f. of the c.d.f. $F(x)$. Now the c.d.f. corresponding to (8) is

$$
G_{F}(x)=I_{F(x)}(\alpha, \beta)
$$

Let $Q_{1}(u)$ be the QF corresponding to $F(x)$, then since c.d.f. and QF are the inverses of each other so using (9) we get the corresponding QF for the transformed variable as

$$
Q_{F}(p)=Q_{1}\left(I_{p}^{-1}(\alpha, \beta)\right)
$$

Hence from Lemma 2 it can be equivalently said that if $Y$ is a beta random variable (r.v.) with QF as given in (5). and if we take a $\mathrm{Q}$-transformation on the random variable $Y$ by an arbitrary $\mathrm{QF} Q_{1}(u)$, then the quantile function for the transformed variable is given by

$$
Q_{F}(p)=Q_{1}\left(I_{p}^{-1}(\alpha, \beta)\right)
$$

The QF defined in (10) denotes the QF of the class of beta generated distribution. The class of beta generated distribution was originally introduced by [15]. The special cases of the QF of this class will be discussed in Section 6.

\section{A Generalized Quantile Function}

McDonald [21] introduced two models of generalized beta distributions as descriptive models for the size distributions of income. The advantages of using such models have been well demonstrated not only by their mathematical tractability but also because these models include many special cases frequently used for size distributions. The generalized beta of the first $(\operatorname{GBI}(a, b, \alpha, \beta))$ and second $(\operatorname{GBII}(a, b, \alpha, \beta))$ kind $[21,22]$ are respectively defined by the p.d.f.s in (11) and (12) and QFs in (13) and (14).

$$
\begin{aligned}
& g(y)=\frac{|a|}{b B(\alpha, \beta)}\left(\frac{y}{b}\right)^{a \alpha-1}\left[1-\left(\frac{y}{b}\right)^{a}\right]^{\beta-1}, 0 \leq y^{a} \leq b^{a} \\
& g(y)=\frac{|a|}{b B(\alpha, \beta)} \frac{\left(\frac{y}{b}\right)^{a \alpha-1}}{\left[1+\left(\frac{y}{b}\right)^{a}\right]^{\alpha+\beta}}, 0 \leq y
\end{aligned}
$$




$$
\begin{aligned}
& Q(p)=b\left[I_{p}^{-1}(\alpha, \beta)\right]^{\frac{1}{a}} \\
& Q(p)=b\left[\frac{I_{p}^{-1}(\alpha, \beta)}{1-I_{p}^{-1}(\alpha, \beta)}\right]^{\frac{1}{a}}
\end{aligned}
$$

where $a \neq 0$ and $b, \alpha$ and $\beta$ are all positive, $b$ is the scale and $a, \alpha, \beta$ are shape parameters.

The constructions of the models (13) and (14) have their origin from model (10) by using the arbitrary QF respectively as the power function distribution, $Q_{1}(p)=b p^{\frac{1}{a}}$ and the Burr XII distribution with one of the shape parameters as unity, $Q_{1}(p)=b\left[(1-p)^{-1}-1\right]^{\frac{1}{a}}$.

The quantile function in (14) can be adjusted to include the quantile function in (13) by including a shape parameter $0 \leq c \leq 1$ such that

$$
Q(p)=b\left[\frac{I_{p}^{-1}(\alpha, \beta)}{1-c I_{p}^{-1}(\alpha, \beta)}\right]^{\frac{1}{a}}
$$

For $c=0$ model (15) reduces to model (13) and for $c=1$ it reduces to model (14)

A distribution encompassing both the GBI and GBII family was proposed by McDonald and Xu [22]. The 5 parameter GB distribution generalizes more than 30 distributions as special and limiting cases. The GB distribution has the potential to yield significantly better fits than any special case. The generalized beta distribution (GB) has the probability density function (p.d.f.)

$$
f(y ; a, b, c, \alpha, \beta)=\frac{|a|}{b B(\alpha, \beta)} \frac{\left(\frac{y}{b}\right)^{a \alpha-1}\left[1-(1-c)\left(\frac{y}{b}\right)^{a}\right]^{\beta-1}}{\left[1+c\left(\frac{y}{b}\right)^{a}\right]^{\alpha+\beta}}, 0 \leq y^{a} \leq \frac{b^{a}}{1-c}
$$

where, $a \neq 0,0 \leq c \leq 1, \alpha>0$ and $\beta>0$ are the shape parameters and $b$ is the scale parameter.

One can interpret about the various parameters of GB distribution from the density plots for different parameter values given in Figure 1.

It can be shown that the quantile function obtained in (15) is the QF of the GB distribution

Proposition 1

The quantile function of a GB distribution is given by

$$
Q(p)= \begin{cases}b\left[\frac{{ }_{l} I_{p}^{-1}(\alpha, \beta)}{1-c_{l} I_{p}^{-1}(\alpha, \beta)}\right]^{\frac{1}{a}}, & \text { if } a>0 \\ b\left[\frac{{ }_{u} I_{p}^{-1}(\alpha, \beta)}{1-c_{u} I_{p}^{-1}(\alpha, \beta)}\right]^{\frac{1}{a}}, & \text { if } a<0\end{cases}
$$

\section{Proof}

To obtain the quantile function of GB, we first need to obtain the distribution function (c.d.f.) of GB. The distributional range is dependent on parameter $a$ which can be both positive and negative. Hence the quantile function has to be defined separately for positive and negative values of $a$. The $c$.d.f. of the standard GB distribution for both positive and negative values of $a$ are: 

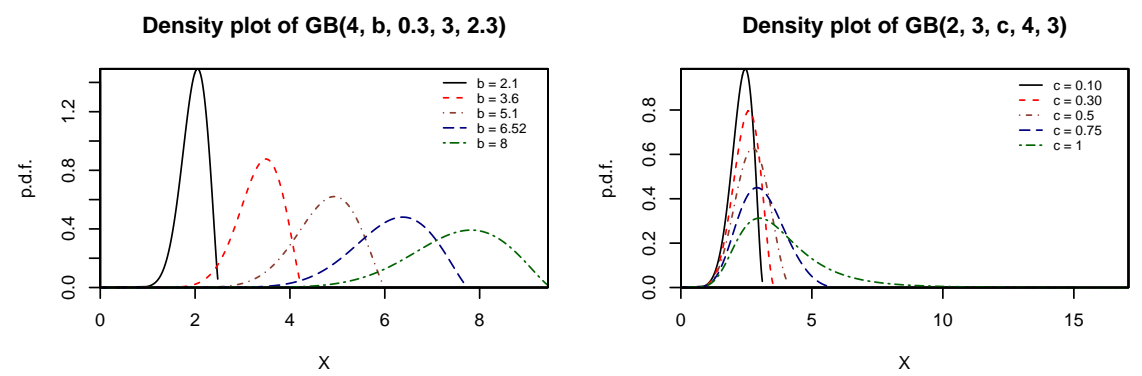

Density plot of GB(a(+)ve, $2,0.5,1.5,2.8)$

Density plot of GB(a(-)ve, $3,0.7,2.2,1.6)$
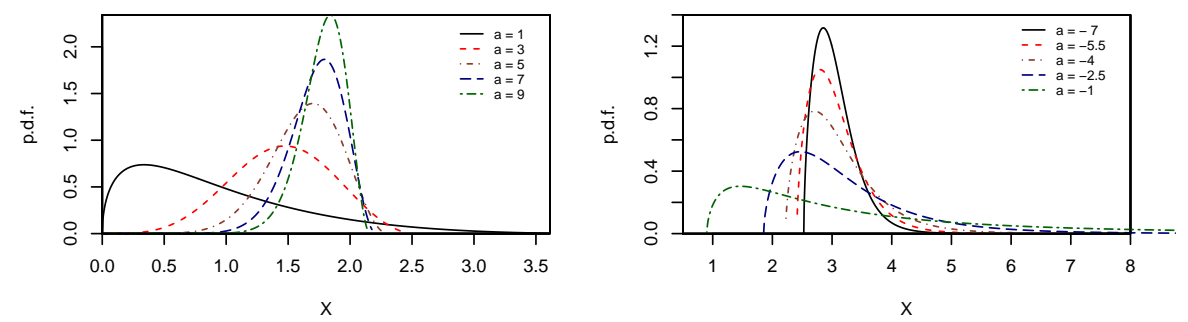

Density plot of $\mathrm{GB}(3.2,5,0.6, \alpha, 4)$

Density plot of $\mathrm{GB}(4,3,0.5,2.8, \beta)$
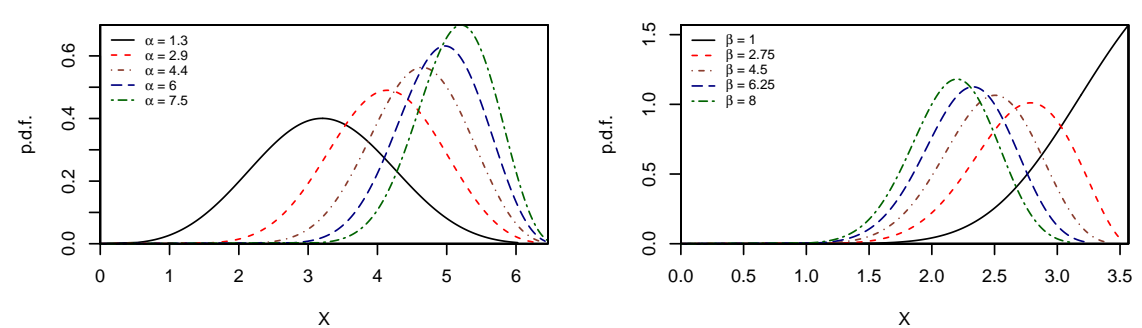

Figure 1. Density plots of GB for various parameter values

$$
\begin{aligned}
& F(x)= \begin{cases}\int_{0}^{x} \frac{|a|}{B(\alpha, \beta)} \frac{y^{a \alpha-1}\left[1-(1-c) y^{a}\right]^{\beta-1}}{\left[1+c y^{a}\right]^{\alpha+\beta}} d y, & \text { if } a>0 \\
\int_{\frac{1}{(1-c)^{1 / a}}}^{x} \frac{|a|}{B(\alpha, \beta)} \frac{y^{a \alpha-1}\left[1-(1-c) y^{a}\right]^{\beta-1}}{\left[1+c y^{a}\right]^{\alpha+\beta}} d y, & \text { if } a<0\end{cases} \\
& = \begin{cases}{ }_{l} I_{\frac{x^{a}}{1+c x^{a}}}(\alpha, \beta), & \text { if } a>0 \\
{ }_{u} I_{\frac{x^{a}}{1+c x^{a}}}(\alpha, \beta), & \text { if } a<0\end{cases}
\end{aligned}
$$

As CDF and QF are inverses of each other, the corresponding quantile function for the standard GB 
distribution is:

$$
Q(p)= \begin{cases}{\left[\frac{{ }_{l} I_{p}^{-1}(\alpha, \beta)}{1-c_{l} I_{p}^{-1}(\alpha, \beta)}\right]^{\frac{1}{a}},} & \text { if } a>0 \\ {\left[\frac{{ }_{u} I_{p}^{-1}(\alpha, \beta)}{1-c_{u} I_{p}^{-1}(\alpha, \beta)}\right]^{\frac{1}{a}},} & \text { if } a<0\end{cases}
$$

This Qf is in the form $S(p, \boldsymbol{\alpha})$. Including the scale parameter $b$ and using lemma 1 , the quantile function for the GB distribution is

$$
Q(p)= \begin{cases}b\left[\frac{{ }_{l} I_{p}^{-1}(\alpha, \beta)}{1-c_{l} I_{p}^{-1}(\alpha, \beta)}\right]^{\frac{1}{a}}, & \text { if } a>0 \\ b\left[\frac{{ }_{u} I_{p}^{-1}(\alpha, \beta)}{1-c_{u} I_{p}^{-1}(\alpha, \beta)}\right]^{\frac{1}{a}}, & \text { if } a<0\end{cases}
$$

The corresponding distribution function involving the scale parameter of GB distribution is

$$
F(x)= \begin{cases}{ }^{l} I_{\frac{(x / b)^{a}}{1+c(x / b)^{a}}}(\alpha, \beta), & \text { if } a>0 \\ { }_{u} I_{\frac{(x / b)^{a}}{1+c(x / b)^{a}}}(\alpha, \beta), & \text { if } a<0\end{cases}
$$

The QF defined in (20) is in the form, $\lambda+\eta S(p, \boldsymbol{\alpha})$, where $\lambda=0, \eta=b$ and $\boldsymbol{\alpha}=(a, c, \alpha, \beta)$. This QF includes the quantile functions of the generalized beta I (GBI) and generalized beta II (GBII) distribution corresponding to $c=0$ and $c=1$, which in turn includes the QF of a numerous distributions as special cases, few of which are shown in table 2.

\section{Theorem 1}

The quantile density function of GB, GBI and GBII distributions are respectively given by (22), (23) and (24)

$$
\begin{aligned}
q(p) & =\frac{b}{a} \frac{I_{p}^{-1}(\alpha, \beta)^{\frac{1}{a}-\alpha}\left(1-I_{p}^{-1}(\alpha, \beta)\right)^{1-\beta} \mathrm{B}(\alpha, \beta)}{\left(1-c I_{p}^{-1}(\alpha, \beta)\right)^{\frac{1}{a}+1}} \\
q(p) & =\frac{b}{a} I_{p}^{-1}(\alpha, \beta)^{\frac{1}{a}-\alpha}\left(1-I_{p}^{-1}(\alpha, \beta)\right)^{1-\beta} \mathrm{B}(\alpha, \beta) \\
q(p) & =\frac{b}{a} I_{p}^{-1}(\alpha, \beta)^{\frac{1}{a}-\alpha}\left(1-I_{p}^{-1}(\alpha, \beta)\right)^{-\beta-\frac{1}{a}} \mathrm{~B}(\alpha, \beta)
\end{aligned}
$$

where $I_{p}^{-1}(\alpha, \beta)$ is the inverse regularized beta function

Proof

The proof is straightforward using the definition of quantile density function.

The density quantile functions given by the reciprocal of the quantile density function can used to obtain the density plot of the generalized beta distribution. 
Table 2. Special Cases of GBI

\begin{tabular}{|c|c|c|}
\hline Special Cases & $Q(p)$ & Distribution Name \\
\hline$\overline{\mathrm{GB}}(1,1,0,1,1)$ & $\overline{p={ }_{l} I_{p}^{-}}$ & Uniform $(0,1)$ \\
\hline $\mathrm{GB}(1, b, 0,1,1)$ & $b p=b_{l} I_{p}^{-1}(1,1)$ & Uniform $(0, b)$ \\
\hline $\operatorname{GB}(1,1,0, \alpha, \beta)$ & ${ }_{l} I_{p}^{-1}(\alpha, \beta)$ & Beta-I \\
\hline $\mathrm{GB}(-1, b, 0, \alpha, 1)$ & $\frac{b}{(1-p)^{\frac{1}{\alpha}}}=b_{u} I_{p}^{-1}(\alpha, 1)$ & Type I Pareto \\
\hline $\operatorname{GB}(-1, b, 0, \alpha, \beta)$ & $b_{u} I_{p}^{-1}(\alpha, \beta)$ & Inverse Beta I \\
\hline $\operatorname{GB}(1, b, 0, \alpha, 1)$ & $b p^{\frac{1}{\alpha}}=b_{l} I_{p}^{-1}(\alpha, 1)$ & Power \\
\hline $\operatorname{GB}(a>0,1,0,1, \beta)$ & {$\left[1-(1-p)^{\frac{1}{\beta}}\right]^{\frac{1}{a}}=\left[{ }_{l} I_{p}^{-1}(1, \beta)\right]^{\frac{1}{a}}$} & Kumaraswamy \\
\hline $\mathrm{GB}(1, b, 1,1,1)$ & $b\left(\frac{p}{1-p}\right)=b\left[\frac{I_{p}^{-1}(1,1)}{1-I_{p}^{-1}(1,1)}\right]$ & Log Logistic \\
\hline $\operatorname{GB}(1, b, 1, \alpha, 1)$ & $b \frac{p^{\frac{1}{\alpha}}}{1-p^{\frac{1}{\alpha}}}=b\left[\frac{I_{p}^{-1}(\alpha, 1)}{1-I_{p}^{-1}(\alpha, 1)}\right]$ & Inverse Lomax \\
\hline $\operatorname{GB}(1, b, 1,1, \beta)$ & $b\left((1-p)^{-\frac{1}{\beta}}-1\right)=b \frac{I_{p}^{-1}(1, \beta)}{1-I_{p}^{-1}(1, \beta)}$ & Lomax \\
\hline $\operatorname{GB}(a>0,1,1,1, \beta)$ & $\left((1-p)^{-\frac{1}{\beta}}-1\right)_{1}^{\frac{1}{a}}=\left[\frac{I_{p}^{-1}(1, \beta)}{1-I_{p}^{-1}(1, \beta)}\right]^{\frac{1}{a}}$ & Singh Maddala \\
\hline $\mathrm{GB}(a>0, b, 1,1, \beta)$ & $b\left((1-p)^{-\frac{1}{\beta}}-1\right)^{\frac{1}{a}}=b\left[\frac{I_{p}^{-1}(1, \beta)}{1-I_{p}^{-1}(1, \beta)}\right]^{\frac{1}{a}}$ & Burr Type XII \\
\hline $\mathrm{GB}(a>0, b, 1, \alpha, 1)$ & $b\left(p^{-\frac{1}{\alpha}}-1\right)^{-\frac{1}{a}}=b\left[\frac{I_{p}^{-1}(\alpha, 1)}{1-I_{p}^{-1}(\alpha, 1)}\right]^{\frac{1}{a}}$ & Burr Type III \\
\hline $\mathrm{GB}(a>0, b, 1,1,1)$ & $b\left(\frac{1-p}{p}\right)^{-\frac{1}{a}}=b\left[\frac{I_{p}^{-1}(1,1)}{1-I_{p}^{-1}(1,1)}\right]^{\frac{1}{a}}$ & Fisk \\
\hline $\operatorname{GB}(1,1,1, \alpha, \beta)$ & $\frac{I_{p}^{-1}(\alpha, \beta)}{1-I_{p}^{-1}(\alpha, \beta)}$ & BetaII \\
\hline $\operatorname{GB}\left(a>0, \frac{\beta}{\alpha}, 1, \frac{\alpha}{2}, \frac{\beta}{2}\right)$ & $\frac{I_{p}^{-1}\left(\frac{\alpha}{2}, \frac{\beta}{2}\right)}{\alpha\left[1-I_{p}^{-1}\left(\frac{\alpha}{2}, \frac{\beta}{2}\right)\right]}$ & F-Distribution \\
\hline
\end{tabular}

\section{Well Known Distributions Obtained by Using the Quantile Functions from GB Family}

The GB distribution covers a large numbers of distributions as its special cases, some of which were mentioned in Table 2. Using some quantile mathematics like the Q-transformation, the p-transformation, addition and multiplication of quantile functions etc., the inter-relation and properties of some wellknown distributions can be obtained from the quantile functions of the GB family. A few such results are discussed below.

1: The reciprocal of the type I Pareto $(b, \alpha)$ distribution is a Power $\left(\frac{1}{b}, \alpha\right)$ distribution.

Illustration: The QF for the type $\mathrm{I} \operatorname{Pareto}(b, \alpha)$ distribution for the variable $X$ is:

$$
Q_{1}(p)=\frac{b}{(1-p)^{\frac{1}{\alpha}}}
$$

The QF for the variable $1 / X$ will be

$$
Q_{2}(p)=\frac{1}{Q_{1}(1-p)}=\frac{1}{b} p^{\frac{1}{\alpha}}
$$

which is the QF for the Power $\left(\frac{1}{b}, \alpha\right)$ distribution. Thus, the power and the Pareto distributions are the inverse of each other.

2: The $\mathrm{QF} Q_{1}(p)$ and $Q_{2}(p)$ discussed above are both positive, hence their product is a valid QF

$$
Q(p)=\left(\frac{p}{1-p}\right)^{\frac{1}{\alpha}}
$$


This is again the QF of the well known Fisk $(1, \alpha)$ distribution.

3: The product of Uniform $(0,1)$ and reciprocal Uniform $(0,1)$ is a $\log$-logistic distribution.

Illustration: $\mathrm{QF}$ for $U(0,1)$ distribution is, $p$. QF for reciprocal $U(0,1)$ distribution is $\frac{1}{1-p}$. The product of these two $\mathrm{QF}$ gives,

$$
Q(p)=\frac{p}{1-p}
$$

the $\mathrm{QF}$ for the log logistic distribution.

4: If $X$ is $\operatorname{arum}(1, \beta)$ distribution [18], then the Q-transformation $-\ln (1-X)$ is an $\operatorname{Exp}(\beta)$ distribution.

Illustration: If $X$ is a $\operatorname{Kum}(1, \beta)$ distribution then its $\mathrm{QF}$ is

$$
Q_{1}(p)=1-(1-p)^{\frac{1}{\beta}}
$$

The QF for the variable $Y=1-X$ is $Q_{2}(p)=1-Q_{1}(1-p)=p^{\frac{1}{\beta}}$. The transformation, $-\ln (Y)$ is a decreasing function of $Y$, hence the corresponding $\mathrm{QF}$ for $-\ln (Y)$ is

$$
Q(p)=-\ln \left(Q_{2}(1-p)\right)=-\ln \left((1-p)^{\frac{1}{\beta}}\right)=-\frac{1}{\beta} \ln (1-p)
$$

which is the QF for the Exponential distribution with parameter $\beta$.

5: If $X$ is $\operatorname{arum}(a, 1)$ distribution [18], then the Q-transformation $-\ln (X)$ is an $\operatorname{Exp}(a)$ distribution. Illustration: Let $X$ is a $\operatorname{Kum}(a, 1)$ distribution, then its $\mathrm{QF}$ is $Q(p)=p^{\frac{1}{a}}$. The $\mathrm{QF}$ for $-\ln (X)$ will be

$$
-\ln (Q(1-p))=-\ln \left((1-p)^{\frac{1}{a}}\right)=-\frac{1}{a} \ln (1-p)
$$

which is the $\operatorname{Exp}(a)$ distribution.

6: The reciprocal of a Weibull $(\alpha, a)$ distribution [34] is a Frechet $\left(\frac{1}{\alpha}, a\right)$ distribution [17, 7 ].

Illustration: The Qf of a Wei $(\alpha, a)$ distribution is

$$
Q(p)=\alpha(-\ln (1-p))^{\frac{1}{a}}
$$

The corresponding reciprocal QF is

$$
\frac{1}{Q(1-p)}=\frac{1}{\alpha(-\ln (p))^{\frac{1}{a}}}=\frac{1}{\alpha}(-\ln (p))^{-\frac{1}{a}}
$$

The Fre $(1 / \alpha, a)$ distribution.

7: A p-transformation, $p^{\frac{1}{a}}$ on the $\operatorname{Kum}(\alpha, \beta)$ distribution transforms it to the exponentiated kumaraswamy (Exp-Kum) distribution [20] with parameters, $a, \alpha$ and $\beta$.

Illustration: The $\mathrm{QF}$ of $\operatorname{Kum}(\alpha, \beta)$ is

$$
Q(p)=\left(1-(1-p)^{\frac{1}{\beta}}\right)^{\frac{1}{\alpha}}
$$

Let $p^{\frac{1}{a}}$ be a p-transformation on $Q(p)$, then the resulting $\mathrm{QF}$ is

$$
\left(1-\left(1-p^{\frac{1}{a}}\right)^{\frac{1}{\beta}}\right)^{\frac{1}{\alpha}}
$$

and this is the QF of the Exp-Kum distribution with $b=1$. 
8: A p-transformation, $p^{\frac{1}{\alpha}}$ on the $\operatorname{Exp}(\lambda)$ distribution transforms it into the exponentiated exponential distribution $[13,14]$ with parameters, $\lambda$ and $\alpha$.

Illustration: The $\mathrm{QF}$ of the $\operatorname{Exp}(\lambda)$ distribution is

$$
Q(p)=-\frac{1}{\lambda} \ln (1-p)
$$

Taking the p-transformation, $p^{\frac{1}{\alpha}}$ on $Q(p)$ we obtain the following $\mathrm{QF}$ as:

$$
-\frac{1}{\lambda} \ln \left(1-p^{\frac{1}{\alpha}}\right)
$$

and this is the QF of the exponentiated exponential distribution with scale parameters $\lambda$ and shape parameter $\alpha$.

9: If $X$ has a $\operatorname{Fisk}(b, \beta)$ distribution, then $Y=\ln (X)$ has a logistic distribution with location $\ln (b)$ and scale $\frac{1}{\beta}$.

Illustration: Let $X \sim \operatorname{Fisk}(b, \beta)$ distribution, then its $\mathrm{QF}$ is

$$
Q(p)=b\left[\frac{p}{1-p}\right]^{\frac{1}{\beta}}
$$

Let $Y=\ln (X)$ be a Q-transformation of the $r . v . X$, then since $Y$ is a non decreasing function of $X$, the QF of $Y$ will be

$$
Q_{Y}(p)=\ln (b)+\frac{1}{\beta} \ln \left(\frac{p}{1-p}\right)
$$

and this is the $\mathrm{QF}$ of a $\operatorname{logistic}\left(\ln (b), \frac{1}{\beta}\right)$ distribution.

10: If $X \sim \operatorname{Par}(b, \alpha)$ distribution then $Y=X-b \sim \operatorname{Lomax}(b, \alpha)$ distribution.

Illustration: Let $X \sim \operatorname{Par}(b, \alpha)$ distribution, then its $\mathrm{QF}$ is

$$
Q(p)=\frac{b}{(1-p)^{\frac{1}{\alpha}}}
$$

Since $Y=X-b$ is a non decreasing transformation of $X$ so its QF will be

$$
Q_{Y}(p)=Q(p)-b=b\left[(1-p)^{-\frac{1}{\alpha}}-1\right]
$$

and this is the QF of the lomax distribution with scale parameters $b$ and shape parameter $\alpha$.

The intermediate distributions discussed above are summarized in table 3.

\section{Size Biased Generalized Beta Distribution}

The concept of weighted distribution was first introduced by Fisher [11] to model ascertainment bias, and was later formalized in a unifying theory by Rao [31]. Let $X$ be a random variable of interest such that $X \sim f(x ; \theta)$, where $\theta$ is a vector of parameters. Then the weighted distribution or the size biased distribution of order $r$ is given by the probability density function in (25).

$$
f_{r}(x, \theta)=\frac{x^{r} f(x, \theta)}{\mu_{r}^{\prime}}
$$


Table 3. Well known Distributions obtained by simple quantile operations

\begin{tabular}{|cccc|}
\hline Dist 1 & Dist 2 & Operations & Resulting Dist \\
\hline \hline $\operatorname{Par}(b, \alpha)$ & & Reciprocal & $\operatorname{Pow}(1 / b, \alpha)=\frac{1}{b} p^{\frac{1}{\alpha}}$ \\
$\operatorname{Par}(b, \alpha)$ & $\operatorname{Pow}(1 / b, \alpha)$ & Product & $\operatorname{Fisk}(1, \alpha)=\left(\frac{p}{1-p}\right)^{\frac{1}{\alpha}}$ \\
$\operatorname{Uni}(0,1)$ & $\operatorname{Reci} \operatorname{Uni}(0,1)$ & Product & $\log -\operatorname{Logistic}=\frac{p}{1-p}$ \\
$\operatorname{Kum}(1, \beta)$ & & Q-trans, $-\ln (1-X)$ & $\operatorname{Exp}(\beta)=-\frac{1}{\beta} \ln (1-p)$ \\
$\operatorname{Kum}(a, 1)$ & Q-trans, $-\ln (X)$ & $\operatorname{Exp}(a)=-\frac{1}{b} \ln (1-p)$ \\
$\operatorname{Wei}(\alpha, a)$ & Reciprocal & $\operatorname{Fre}(1 / \alpha, a)=\frac{1}{\alpha}(-\ln (p))^{-\frac{1}{a}}$ \\
$\operatorname{Kum}(\alpha, \beta)$ & p-trans, $p^{\frac{1}{a}}$ & $\operatorname{Exp}-\operatorname{Kum}(a, b=1, \alpha, \beta)=\left(1-\left(1-p^{\frac{1}{a}}\right)^{\frac{1}{\beta}}\right)^{\frac{1}{\alpha}}$ \\
$\operatorname{Exp}(\lambda)$ & p-trans, $p^{\frac{1}{\alpha}}$ & $\operatorname{Exp}-\operatorname{EXP}(\lambda, \alpha)=-\frac{1}{\lambda} \ln \left(1-p^{\frac{1}{\alpha}}\right)$ \\
$\operatorname{Fisk}(b, \beta)$ & Q-trans, $\ln (X)$ & $\operatorname{Logistic}\left(\ln (b), \frac{1}{\beta}\right)=\ln (b)+\frac{1}{\beta} \ln \left(\frac{p}{1-p}\right)$ \\
$\operatorname{Par}(b, \alpha)$ & & Q-trans, $X-b$ & $\operatorname{Lomax}(b, \alpha)=b\left[(1-p)^{-\frac{1}{\alpha}}-1\right]$ \\
\hline
\end{tabular}

where

$$
\mu_{r}^{\prime}=\int x^{r} f(x ; \theta) d x
$$

It can be shown that closed form expression of the quantile function of size-biased generalized beta distribution doesn't exist and so we study and obtain the quantile functions of size biased GBI and GBII distributions.

Lemma 3

Let the quantile functions of GBI and GBII as given in (13) and (14) be denoted respectively by $Q_{I}(p)$ and $Q_{I I}(p)$, then the quantile functions of the weighted distribution of the GBI and GBII are respectively given by (26) and (27).

$$
\begin{gathered}
Q_{S B I}(p)= \begin{cases}b\left[{ }_{l} I_{p}^{-1}(\alpha+r / a, \beta)\right]^{\frac{1}{a}}, & \text { if } a>0 \\
b\left[{ }_{u} I_{p}^{-1}(\alpha+r / a, \beta)\right]^{\frac{1}{a}}, & \text { if } a<0\end{cases} \\
Q_{S B I I}(p)= \begin{cases}b\left[\frac{{ }_{l} I_{p}^{-1}(\alpha+r / a, \beta-r / a)}{1-{ }_{l} I_{p}^{-1}(\alpha, \beta)}\right]^{\frac{1}{a}}, & \text { if } a>0 \\
b\left[\frac{{ }_{u} I_{p}^{-1}(\alpha+r / a, \beta-r / a)}{1-{ }_{u} I_{p}^{-1}(\alpha, \beta)}\right]^{\frac{1}{a}}, & \text { if } a<0\end{cases}
\end{gathered}
$$

\section{Proof}

Ducey and Gove [9] have obtained the weighted distribution of the $\operatorname{GBI}(a, b, \alpha, \beta)$ and the $\operatorname{GBII}(a, b, \alpha, \beta)$ distributions and have shown that the GBI and the GBII distributions are form invariant under size biased scheme for all orders $r$ for which the $r^{\text {th }}$ order moments exist; i.e., the size-biased distribution of order $r$ or the weighted distribution has the same mathematical form as the original distribution, with different parameter values. Accordingly, the weighted distribution of GBI with parameters $a, b, \alpha$ and $\beta$ is a GBI with parameters $a, b, \alpha+r / a$ and $\beta$, and the weighted distribution of GBII with parameters $a, b, \alpha$ and $\beta$ is a GBII with parameters $a, b, \alpha+r / a$ and $\beta-r / a$. Hence using relation (13) and (14), the proof follows. 
Thus, the quantile function of the weighted distribution of any distribution in the generalized beta family can be obtained directly using Lemma 3. The Kumaraswamy distribution [18] is a distribution in the GBI family of distributions with parameters, $a, 1,1$, and $\beta=b$. Hence using Lemma 3, a new distribution viz., the Size Biased Kumaraswamy distribution (SBKD) [32] have been obtained and is given by the quantile function

$$
Q(p)=\left[I_{p}^{-1}\left(1+\frac{1}{a}, b\right)\right]^{\frac{1}{a}}
$$

where, $a$ and $b$ are the two shape parameters. For a detailed study on the properties and applications of SBKD one can refer to [32].

\section{Beta generated distributions}

Beta normal distribution, a special case of the class of beta generated (BG) distributions have been first proposed by [10]. The class of beta generated distributions was then made prominent by [15]. The quantile function for this class of distribution is given in (29) by

$$
Q_{F}(p)=Q_{1}\left(I_{p}^{-1}(\alpha, \beta)\right)
$$

where $Q_{1}($.$) is an arbitrary QF. The derivation for (29) has been shown in Lemma 2. This quantile function$ shall be termed as the beta generated quantile function (BGQF) as it has its origin in the distribution of a beta random variable $Y$ with $\mathrm{QF}$

$$
Q_{b}(p)=I_{p}^{-1}(\alpha, \beta)
$$

for $\alpha, \beta>0$. The BGQF can be used to obtain quantile functions of other well known distributions in the BG class. Some of which are discussed below

1. Beta normal distribution [10]: The QF for the beta normal (BN) distribution can be obtained by using the arbitrary QF in (29) as the QF of the normal distribution, i.e., $Q_{1}(u)=\mu+\sigma \Phi^{-1}(u)$. Thus the $\mathrm{QF}$ for the $\mathrm{BN}$ distribution is given by

$$
\begin{aligned}
Q_{B N}(p) & =Q_{1}\left(I_{p}^{-1}(\alpha, \beta)\right) \\
& =\mu+\sigma \Phi^{-1}\left(\left[I_{p}^{-1}(\alpha, \beta)\right]\right)
\end{aligned}
$$

It can be proved that (30) is the $\mathrm{QF}$ of the beta normal distribution.

2. Beta exponential distribution [27]: The QF for the beta exponential (BE) distribution can be obtained by using the arbitrary QF in (29) as the QF of the exponential distribution, i.e., $Q_{1}(u)=-\frac{1}{\lambda} \ln (1-u)$. Thus the $\mathrm{QF}$ for the $\mathrm{BE}$ distribution is given by

$$
Q_{B E}(p)=-\frac{1}{\lambda}\left(\ln \left[1-I_{p}^{-1}(\alpha, \beta)\right]\right)
$$

3. Beta gumbel distribution [26]: Using the arbitrary QF in (29) as the QF of the gumbel distribution, i.e., $Q_{1}(u)=\mu-\sigma \ln (-\ln (u))$, we obtain the $\mathrm{QF}$ for the beta gumbel (BG) distribution. Thus the $\mathrm{QF}$ for the $\mathrm{BG}$ distribution is given by

$$
Q_{B G}(p)=\mu-\sigma \ln \left(-\ln \left[I_{p}^{-1}(\alpha, \beta)\right]\right)
$$

Similarly, we can obtain the QF of many other beta generated distributions like the Beta weibull distribution [19], Beta pareto distribution [2], Beta Rayleigh distribution [1], Beta Fréchet distribution [24], Beta burr distribution [29], Beta dagum distribution [8] and Beta half logistic distribution [16] etc. 
The QF of these distributions are shown in Table 4. Let us now take an exponentiated p-transformation of $\frac{1}{a}$ on the baseline quantile function, i.e. the beta QF. Then using the p-transformation rule we obtain a class of distribution having the $\mathrm{QF}$ in (33).

$$
Q_{F}(p)=Q_{1}\left(\left[I_{p}^{-1}(\alpha, \beta)\right]^{\frac{1}{a}}\right)
$$

It can be proved that the QF as obtained in (33) is the QF of the class of generalized beta generated distribution (GBGQF) [3].

Lemma 4

Let $Y$ be a generalized beta I random variable (r.v.) with $\mathrm{QF} Q(p)=\left[I_{p}^{-1}(\alpha, \beta)\right]^{\frac{1}{a}}$. If we take a Qtransformation on the random variable $Y$ by an arbitrary $\mathrm{QF} Q_{1}(u)$, then the quantile function for the transformed variable is given by

$$
Q_{F}(p)=Q_{1}\left(\left[I_{p}^{-1}(\alpha, \beta)\right]^{\frac{1}{a}}\right)
$$

Proof

Lemma 4 can be proved using the similar approach as Lemma 2.

Special case The GBGQF has the following main sub cases

1. For $a=1$, the class of GBGQF is the class of BGQF as given in (29)

2. For $\alpha=1$, the class of GBGQF is the class of Kumaraswamy generated quantile function (KGQF) [[6]] given by

$$
Q_{F}(p)=Q_{1}\left(\left[1-(1-p)^{\frac{1}{\beta}}\right]^{\frac{1}{a}}\right)
$$

3. For $a=\beta=1$, the class of GBGQF is the class of exponentiated distributions (EQF) with an exponentiated p-transformation of $\frac{1}{\alpha}$ on the baseline QF and is given by

$$
Q_{F}(p)=Q_{1}\left(p^{\frac{1}{\alpha}}\right)
$$

(36) is the quantile form for the Lehman type I distributions.

4. For $a=\alpha=1$, the class of GBGQF is the class of exponentiated distributions (EQF) with a reverse exponentiated $\mathrm{p}$-transformation of $\frac{1}{\beta}$ on the baseline $\mathrm{QF}$ and is given by

$$
Q_{F}(p)=Q_{1}\left(1-(1-p)^{\frac{1}{\beta}}\right)
$$

(37) is the quantile form for the Lehman type II distributions.

Hence the class of EQF comprises of distributions with exponentiated and reverse exponentiated ptransformations of the baseline distribution. The class of EQF contains the QF of distributions like, the exponentiated weibull (EW) distribution [23], the exponentiated exponential (EE) distribution [14], the exponentiated gumbel (EG) distribution [25], the exponentiated Kumaraswamy distribution [20], the exponentiated Fréchet distribution [28] etc. The sub-models of the sub cases of GBGQF are presented in Table 4 
Table 4. QF of well known distributions generated using sub classes of GBGQF

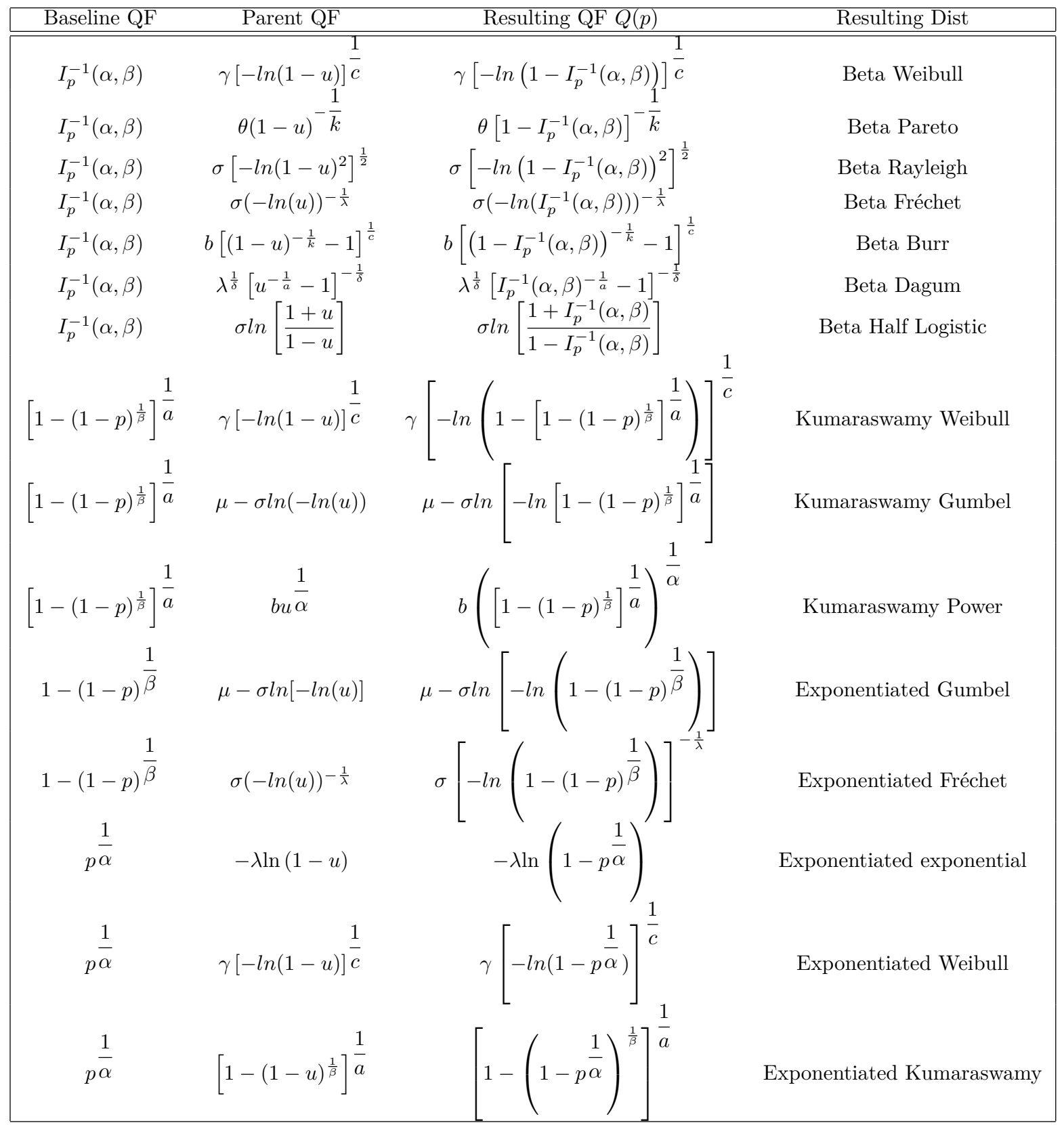

\section{Applications}

\section{Quantile based GBG distributions}

The class of generalized beta generated (GBG) distribution has been proposed much earlier by [3] and has applications in generating new distributions in classical form, i.e. by choosing a classical distribution as the arbitrary parent distribution. Suppose that instead of choosing a classical distribution we are 
interested in choosing a quantile based distribution as the parent distribution, then the quantile form of GBG distribution is the only alternative. The quantile form of the GBG distribution provides flexibility in generating not only the classical GBG distributions but also quantile based GBG distributions and this provides one of the most important applications of the class of GBGQF. As an example let us consider the quantile based skew logistic distribution (SLD) [33], which is given by

$$
Q_{S L D}(p)=\alpha+\beta[(1-\delta) \ln (p)-\delta \ln (1-p)]
$$

Suppose now using this model as the parent distribution and the EQF model in (36) as the baseline distribution we generate a new model. Then the new model, i.e. Exponentiated quantile based skew logistic distribution $\left(\mathrm{ESLD}_{Q B}\right)$ is given by

$$
Q_{S L D}(p)=\alpha+\beta\left[(1-\delta) \ln \left(p^{\frac{1}{\alpha}}\right)-\delta \ln \left(1-p^{\frac{1}{\alpha}}\right)\right]
$$

The exponentiated quantile based SLD obtained in model (39) is same as the generalized quantile based SLD proposed by [4] with slight reparameterization of $\alpha=1 / \kappa$. Thus, valid quantile based distributions can be generated using the generating quantile function in (33) and its various sub cases defined so far.

\section{Density plots}

The quantile density function of GBGQF is given by

$$
q(p)=\frac{1}{a} \mathrm{~B}(\alpha, \beta) I_{p}^{-1}(\alpha, \beta)^{\frac{1}{a}-\alpha}\left(1-I_{p}^{-1}(\alpha, \beta)\right)^{1-\beta} q_{1}\left(I_{p}^{-1}(\alpha, \beta)^{\frac{1}{a}}\right)
$$

where $q_{1}($.$) is the quantile density function corresponding to the arbitrary parent QF. The density quantile$ function of the GBGQF given by the reciprocal of the quantile density function defined in (40) can be used to plot the p.d.f. of the distributions belonging to this class which will give us an idea of the shape of the density curves of this class. As an example we consider the BE and EE distributions, both of which belong to the GBG class. Using the density quantile function, p.d.f. of BE and EE have been plotted and as a means of verification the p.d.f. of BE and EE have been replotted using R package "Newdistns". If the density plot using (40) is valid, then it should coincide with the density plotted by using "Newdistns". Figure 2 shows density plots of BE and EE using the density quantile function and "Newdistns", and it clearly proves that density plotted by using QF is equivalent to density plotted by using the density function. Hence GBGQF has applications in plotting p.d.f. of distributions belonging to this class.

\section{Random number generation}

The GBGQF can be used to generate random samples of any size from the distributions belonging to this class by using uniform transformation rule. Let $U$ be a uniform $(U(0,1)) r \cdot v$. and let $Q(p), 0 \leq p \leq 1$ be the quantile function of the beta generated distribution as defined in (33), then by uniform transformation rule, the variable $X$, where $x=Q(u)$, has a distribution with quantile function $Q(p)$.

As an example let us consider two generalized beta generated distributions, viz., the Beta Frechet distribution (BF) and the exponentiated skew logistic distribution (ESLD). [5] have successfully fitted the $\mathrm{BF}$ distribution to the breaking stress of carbon fibre data containing 100 observations by the method of maximum likelihood estimation (MLE). The estimates so obtained are $\widehat{\alpha}=0.4108, \widehat{\beta}=125.1891, \widehat{\lambda}=$ 0.7496 and $\widehat{\sigma}=31.4556$. Considering these values as known parameters we simulate a random sample of size 100 from the BF distribution and obtain the density plot and Q-Q plot for the same and compare it with the density and Q-Q plot of the fitted distribution as shown in Figure 3.

Similarly the estimates for the ESLD obtained by the method of matching L-moments in the work by [4] in successfully fitting PCB concentration data are used to simulate random sample from ESLD. The 
Density plot of Beta Exponential distribution

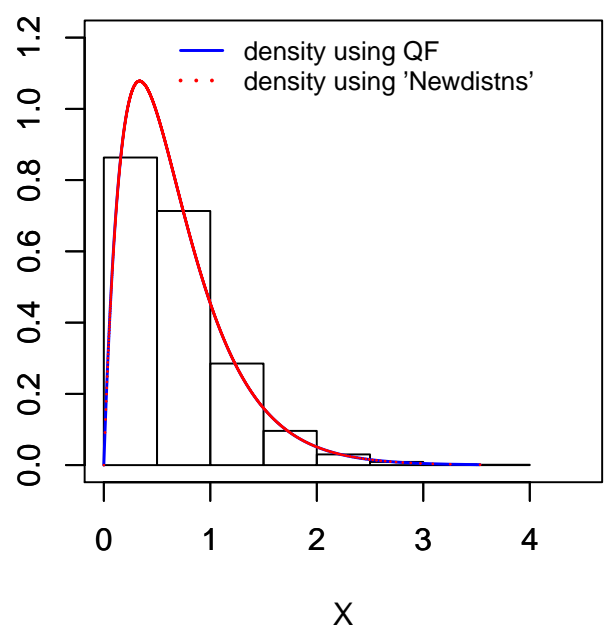

Density plot of Exponentiated Exponential distribution

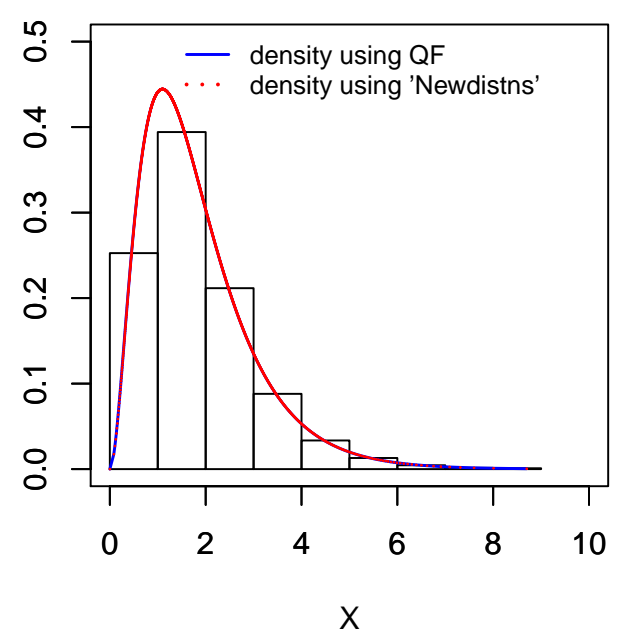

Figure 2. Density plot of BE and EE using QF and "Newdistns"
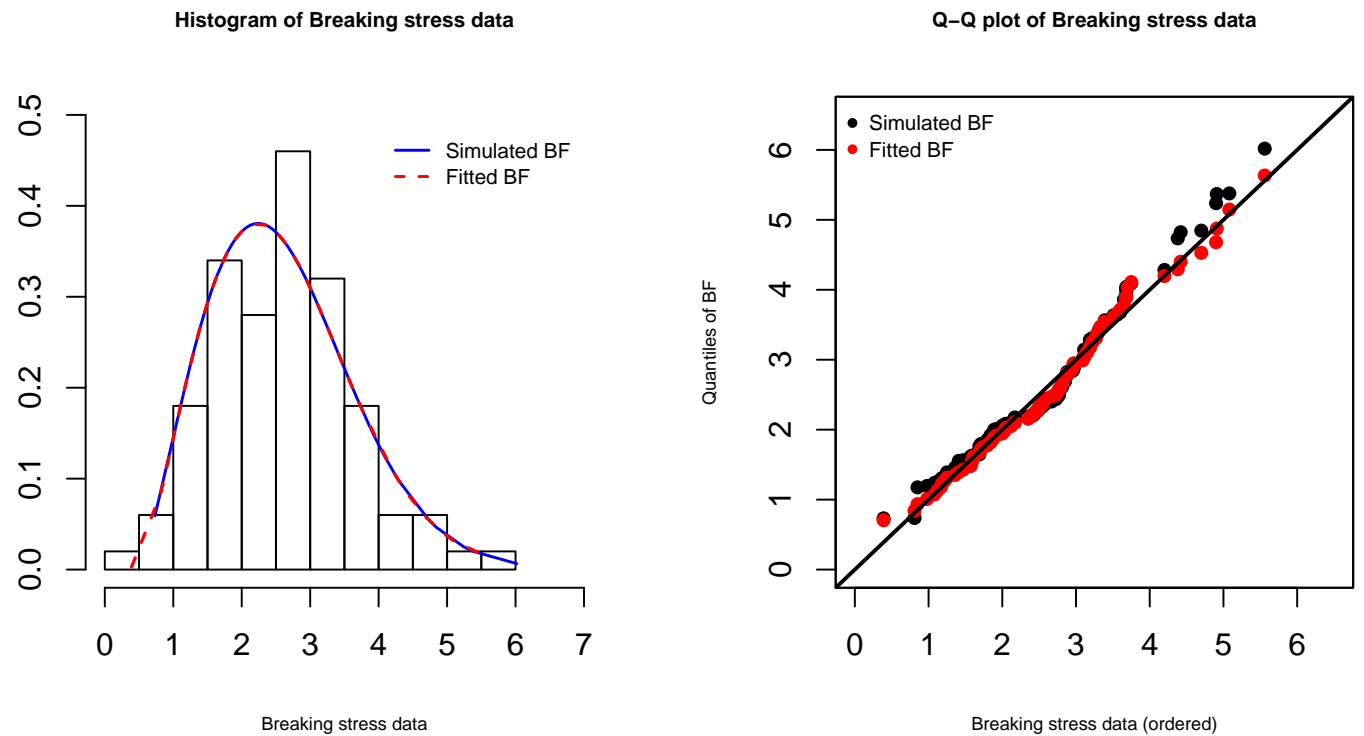

Figure 3. Density and QQ plot of both simulated and fitted Breaking Stress data

estimates were obtained as $\widehat{\alpha}=218.95, \widehat{\beta}=76.240, \widehat{\delta}=0.81592$ and $\widehat{\kappa}=2.7538$. The density plot and Q-Q-plot for the simulated sample and the fitted values have been shown in Figure 4.

In both the figures it can be seen that the simulated data gives a good fit to the original data clearly indicating the application of quantile function in simulation study. Thus, by using quantile function of a distribution in particular, the generalized beta family of distributions, one can generate a random sample 

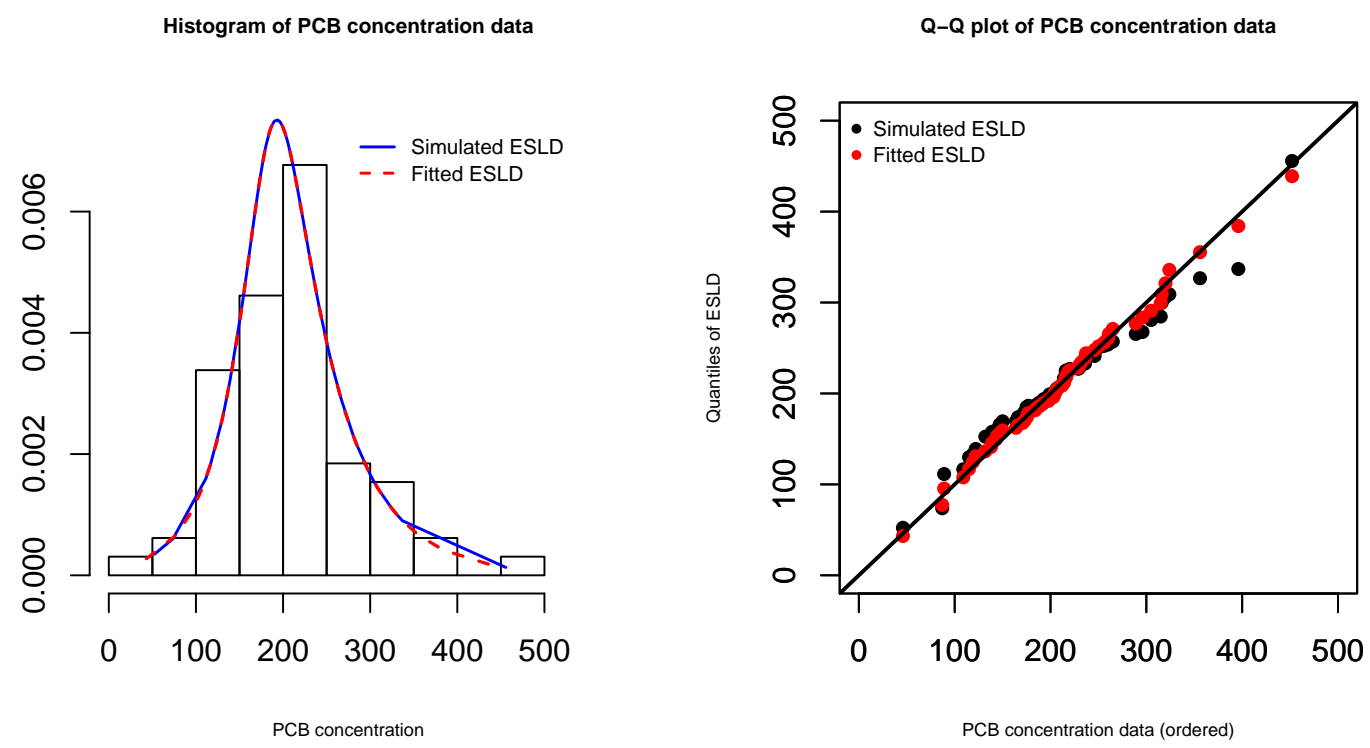

Figure 4. Density and QQ plot of both simulated and fitted PCB Concentration data

of a desired size from that distribution and make a study to obtain or discuss the properties, inference and various related results.

\section{Conclusion}

The paper showed the use of Quantile functions to study a generalized family; viz, the GB family and some general results of the family have been derived by fully using QFs. The inter relationship and/or properties of some of the distributions under the family have been shown using quantile approach such as the Q-transformation, the $p$-transformation, addition and multiplication of quantile functions etc. It has also been shown how using QF of the GBI and GBII distributions the corresponding size biased versions can be obtained. The QF of beta distribution has also been used to derive the class of beta generated distribution and generalized beta generated distribution in quantile form. The QF of beta generated, kumaraswamy generated and exponentiated distributions have been obtained as sub models of GBGQF. QF of some well known distributions belonging to these sub models have been obtained. The results derived in this article are successfully applied to real and simulated datasets, which demonstrate the importance and effectiveness of QF in statistical modelling.

Acknowledgments: The first author acknowledges the Department of Science and Technology (DST), Government of India for her financial support through DST-INSPIRE fellowship with award no. IF130343. The authors thank the anonymous reviewers for their valuable comments. 
1. A. Akinsete, and C. Lowe, Beta-rayleigh distribution in reliability measure. Section on physical and engineering sciences, Proceedings of the American Statistical Association, vol. 1, pp. 3103-3107, 2009.

2. A. Akinsete, F. Famoye, and C. Lee, The beta-pareto distribution Statistics, vol. 42, no. 6, pp. 547-563, 2008.

3. C. Alexander, G.M. Cordeiro, E.M.M. Ortega, and J.M. Sarabia, Generalized beta-generated distributions, Computational Statistics \& Data Analysis, vol. 56, no. 6, pp. 1880-1897, 2012.

4. N. Balakrishnan, and H.Y. So, A generalization of quantile-based skew logistic distribution of van staden and king, Statistics \& Probability Letters, vol. 107, pp. 44-51, 2015.

5. W. Barreto-Souza, G.M. Cordeiro, and A.B. Simas, Some results for beta fréchet distribution, Communications in Statistics: Theory and Methods, vol. 40, no. 5, pp. 798-811, 2011.

6. G.M. Cordeiro, and M. De Castro, A new family of generalized distributions, Journal of statistical computation and simulation, vol. 81, no. 7, pp. 883-898, 2011.

7. Felipe RS De Gusmão, E.M.M. Ortega, and G.M. Cordeiro, The generalized inverse weibull distribution, Statistical Papers, vol. 52, no. 3, pp. 591-619, 2011.

8. F. Domma, and F. Condino, The beta-dagum distribution: definition and properties, Communications in Statistics: Theory and Methods, vol. 42, no. 22, pp. 4070-4090, 2013.

9. M.J. Ducey, and J.H. Gove, Size-biased distributions in the generalized beta distribution family, with applications to forestry, Forestry, vol. 81, no. 1, pp. 143-151, 2015.

10. N. Eugene, C. Lee, and F. Famoye, Beta-normal distribution and its applications, Communications in Statistics: Theory and methods, vol. 31, no. 4, pp. 497-512, 2002.

11. R.A. Fisher, The effect of methods of ascertainment upon the estimation of frequencies, Annals of Human Genetics, vol. 6, no. 1, pp. 13-25, 1934.

12. W.G. Gilchrist, Statistical modelling with quantile functions, Chapman and Hall, New York, 2000.

13. R.D. Gupta, and D. Kundu, Theory 89 methods: Generalized exponential distributions, Australian \& New Zealand Journal of Statistics, vol. 41, no. 2, pp. 173-188, 1999.

14. R.D. Gupta, and D. Kundu, Exponentiated exponential family: an alternative to gamma and weibull distributions, Biometrical journal, vol. 43, no. 1, pp. 117-130, 2001.

15. M.C. Jones, Families of distributions arising from distributions of order statistics, Test, vol. 13, no. 1, pp. 1-43, 2004.

16. J.K. Jose, and M Manoharan, Beta half logistic distribution-a new probability model for lifetime data, Journal of Statistics and Management Systems, vol. 19, no. 4, pp. 587-604, 2016.

17. M.S. Khan, G.R. Pasha, and A.H. Pasha, Theoretical analysis of inverse weibull distribution, WSEAS Transactions on Mathematics, vol. 7, no. 2, pp. 30-38, 2008.

18. P. Kumaraswamy, A Generalized probability density function for double-bounded random processes, Journal of Hydrology, vol. 46, no. 1, pp. 79-88, 1980.

19. C. Lee, F. Famoye, and O. Olumolade, Beta-weibull distribution: some properties and applications to censored data, Journal of modern applied statistical methods, vol. 6, no. 1, pp. 17, 2007.

20. A.J. Lemonte, W. Barreto-Souza, and G.M. Cordeiro, The exponentiated kumaraswamy distribution and its logtransform, Brazilian Journal of Probability and Statistics, vol. 27, no. 1, pp. 31-53, 2013.

21. J. B. McDonald, Some generalized functions for the size distribution of income, Econometrica, vol. 52, no. 3, pp. 647-663, 1984.

22. J.B. McDonald, and Y.J. Xu, A generalization of the beta distribution with applications, Journal of Econometrics, vol. 66 , no. 1 , pp. 133-152, 1995.

23. G.S. Mudholkar, D.K. Srivastava, and M. Freimer, The exponentiated weibull family: a reanalysis of the bus-motorfailure data, Technometrics, vol. 37, no. 4, pp. 436-445, 1995.

24. S. Nadarajah, and A.K. Gupta, The beta fréchet distribution, Far East Journal of Theoretical Statistics, vol. 14, no. 1 , pp. $15-24,2004$.

25. S. Nadarajah, The exponentiated gumbel distribution with climate application, Environmetrics, vol. 17, no. 1, pp. $13-23,2006$

26. S. Nadarajah, and S. Kotz, The beta gumbel distribution, Mathematical Problems in Engineering, vol. 2004, no. 4 , pp. 323-332, 2004.

27. S. Nadarajah, and S. Kotz, The beta exponential distribution, Reliability engineering \& system safety, vol. 91, no. 6, pp. 689-697, 2006.

28. S. Nadarajah, and S. Kotz, The exponentiated type distributions, Acta Applicandae Mathematica, vol. 92, no. 2, pp. 97-111, 2006.

29. P.F. Paranaíba, E.M.M. Ortega, G.M. Cordeiro, and R.R. Pescim, The beta burr xii distribution with application to lifetime data, Computational Statistics \& Data Analysis, vol. 55, no. 2, pp. 1118-1136, 2011.

30. E. Parzen, Quantile probability and statistical data modeling, Statistical Science, pp. 652-662, 2004.

31. C.R. Rao, On discrete distributions arising out of methods of ascertainment, In G.P. Patil, editor, Classical and Contagious Discrete Distributions, pp. 320-332. Pergamon Press and Statistical Publishing Society, Calcutta, 1965.

32. D. Sharma, and T.K. Chakrabarty, On size biased kumaraswamy distribution Statistics, Optimization and Information Computing, vol. 4, no. 3, pp. 252-264, 2016.

33. P.J. van Staden, and Robert A.R. King, The quantile-based skew logistic distribution, Statistics \& Probability Letters, vol. 96, pp. 109-116, 2015.

34. W. Weibull, A statistical distribution function of wide applicability, Journal of Applied Mechanics-Transactions of the Asme, vol. 18, no. 3, pp. 293-297, 1951. 